\title{
Crystal structure of catena-poly[dichlorido- $\left(\mu_{2}-4-\right.$ (1H-pyrazol-3-yl)-pyridine- $\left.\left.\mathrm{K}^{2} N, N^{\prime}\right)\right]$ cadmium(II), $\mathrm{C}_{48} \mathrm{H}_{42} \mathrm{Cd}_{3} \mathrm{Cl}_{16} \mathrm{~N}_{18}$
}

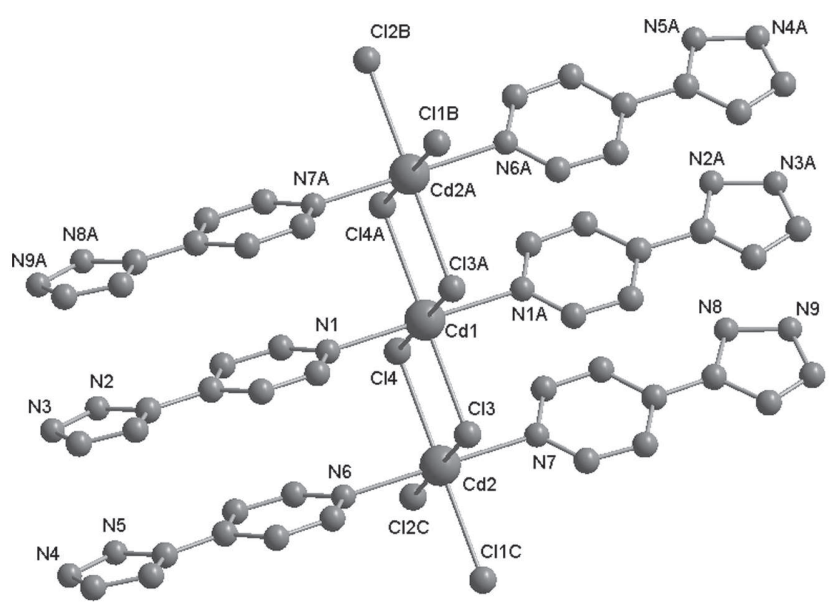

DOI 10.1515/ncrs-2016-0363

Received December 26, 2016; accepted June 14, 2017; available online June 28, 2017

\section{Abstract \\ $\mathrm{C}_{48} \mathrm{H}_{42} \mathrm{Cd}_{3} \mathrm{Cl}_{16} \mathrm{~N}_{18}$, monoclinic, $C 2 / c$ (no. 15), $a=11.4564(7) \AA$, $b=21.1547(15) \AA, \quad c=21.3475(13) \AA, \quad \beta=95.192(5)^{\circ}$, $V=5152.5(6) \AA^{3}, Z=4, R_{\mathrm{gt}}(F)=0.0381, w R_{\text {ref }}\left(F^{2}\right)=0.0862$, $T=293(2) \mathrm{K}$.}

\section{CCDC no.: 1555765}

Parts of the title crystal structure are shown in the figure. Tables 1 and 2 contain details of the measurement method and a list of the atoms including atomic coordinates and displacement parameters.

\section{Source of material}

A mixture of $\mathrm{Cd}\left(\mathrm{NO}_{3}\right)_{2} \cdot 2 \mathrm{H}_{2} \mathrm{O}(0.1 \mathrm{mmol}, 0.0272 \mathrm{~g})$, 4-(1Hpyrazol-3-yl)-pyridine (0.1 mmol, $173.2 \mathrm{mg}), \mathrm{HCl}(2 \mathrm{~mL})$ and distilled water $(8 \mathrm{~mL})$ was heated in a $25 \mathrm{~mL}$ stainless steel

*Corresponding author: Ruo-Fei Bai, College of Chemistry and Chemical Engineering, Luoyang Normal University, Luoyang 471022, Henan Province, P. R. China, e-mail: shihui471022@126.com Qiu-Xia Liu: College of Chemistry and Chemical Engineering, Luoyang Normal University, Luoyang 471022, Henan Province, P. R. China
Table 1: Data collection and handling.

\begin{tabular}{ll}
\hline Crystal: & Colourless block \\
Size: & $0.42 \times 0.32 \times 0.21 \mathrm{~mm}$ \\
Wavelength: & Mo $K \alpha$ radiation $(0.71073 \AA)$ \\
$\mu:$ & $29.3 \mathrm{~cm}^{-1}$ \\
Diffractometer, scan mode: & Bruker Multiwire, $\varphi$ and $\omega$ \\
$2 \theta_{\text {max }}$, completeness: & $51^{\circ},>99 \%$ \\
$N(h k l)_{\text {measured }}, N(h k l)_{\text {unique }}, R_{\text {int }}:$ & $14107,4780,0.028$ \\
Criterion for $I_{\text {obs }}, N(h k l)_{\text {gt }}:$ & $I_{\text {obs }}>2 \sigma\left(I_{\text {obs }}\right), 1412$ \\
$N(\text { param })_{\text {refined }}:$ & 340 \\
Programs: & Bruker programs [1], SHELX [2] \\
\hline
\end{tabular}

reactor with a teflon liner at $443 \mathrm{~K}$ for $38 \mathrm{~h}$, followed by slow cooling to room temperature. Colourless crystals of the compound were formed.

\section{Experimental details}

All $\mathrm{H}$ atoms were positioned geometrically $(\mathrm{N}-\mathrm{H}=0.86 \AA$ and $\mathrm{C}-\mathrm{H}=0.93 \AA$ ) and refined using a riding model, with $U_{\text {iso }}(\mathrm{H})=1.2 U_{\text {eq }}(\mathrm{C}, \mathrm{N})$.

\section{Comment}

Framework materials based on self assembly of organic ligands and coordinated metal ions are the subject of intense research activity and have found applications in the fields of gas storrage [3], catalysis [4], magnetism [5] and luminescence [6]. Many efforts have been devoted to use of $\mathrm{N}$ - and $\mathrm{O}$-donor organic ligands as co-ligands to bridge metal ions to form infinite network structures, such as one-dimensional (1D) chain structures and 2- or 3-D networks [7, 8]. However, the design of predictable frameworks with desired properties remains a considerable challenge.

The data, collected at room temperature, indicate the presence of whole-molecule disorder, with each of the three pyrazol rings contained in the asymmetric unit disordered about the approximate, molecular (non-crystallographic) two-fold rotation axes of the pyridine rings. Disorder could not be satisfactorily modelled and an ordered model is presented here. 
Table 2: Fractional atomic coordinates and isotropic or equivalent isotropic displacement parameters $\left(\AA^{2}\right)$.

\begin{tabular}{|c|c|c|c|c|}
\hline Atom & $x$ & $y$ & $z$ & $U_{\text {iso }} * / U_{\text {eq }}$ \\
\hline Cd1 & 0.0000 & $0.12501(4)$ & 0.7500 & $0.0312(2)$ \\
\hline $\mathrm{Cd} 2$ & $0.33342(5)$ & $0.12499(3)$ & $0.74998(3)$ & $0.03094(16)$ \\
\hline $\mathrm{Cl} 1$ & 0.5000 & $0.03602(13)$ & 0.7500 & $0.0328(6)$ \\
\hline $\mathrm{Cl} 2$ & 0.5000 & $0.21384(13)$ & 0.7500 & $0.0343(7)$ \\
\hline $\mathrm{Cl} 3$ & $0.16720(16)$ & $0.03600(8)$ & $0.75010(9)$ & $0.0319(4)$ \\
\hline $\mathrm{Cl} 4$ & $0.16681(15)$ & $0.21399(8)$ & $0.75000(9)$ & $0.0321(4)$ \\
\hline N1 & $-0.0185(5)$ & $0.1245(3)$ & $0.6409(3)$ & $0.0322(16)$ \\
\hline N2 & $-0.0270(6)$ & $0.1772(4)$ & $0.4038(4)$ & $0.075(3)$ \\
\hline N3 & $-0.0517(8)$ & $0.1529(8)$ & $0.3435(4)$ & $0.131(6)$ \\
\hline H3 & -0.0467 & 0.1736 & 0.3091 & $0.157^{\star}$ \\
\hline N4 & $0.2830(8)$ & $0.1539(7)$ & $0.3459(5)$ & $0.123(5)$ \\
\hline $\mathrm{H} 4$ & 0.2920 & 0.1753 & 0.3124 & $0.148^{\star}$ \\
\hline N5 & $0.3047(6)$ & $0.1764(5)$ & $0.4064(4)$ & $0.076(3)$ \\
\hline N6 & $0.3163(5)$ & $0.1258(3)$ & $0.6418(2)$ & $0.0308(16)$ \\
\hline N7 & $0.3520(5)$ & $0.1247(3)$ & $0.8582(3)$ & $0.0298(15)$ \\
\hline N8 & $0.3631(6)$ & $0.1774(4)$ & $1.0954(4)$ & $0.079(3)$ \\
\hline N9 & $0.3843(9)$ & $0.1527(8)$ & $1.1553(5)$ & $0.135(6)$ \\
\hline H9 & 0.3763 & 0.1728 & 1.1896 & $0.162^{\star}$ \\
\hline C1 & $0.4186(7)$ & $0.0740(5)$ & $1.0949(5)$ & $0.061(3)$ \\
\hline $\mathrm{H} 1$ & 0.4369 & 0.0340 & 1.0805 & $0.074^{\star}$ \\
\hline C2 & $0.3848(6)$ & $0.1260(4)$ & $1.0577(3)$ & $0.0333(19)$ \\
\hline C3 & $0.4201(9)$ & $0.0909(8)$ & $1.1514(6)$ & $0.088(5)$ \\
\hline $\mathrm{H} 3 \mathrm{~A}$ & 0.4423 & 0.0653 & 1.1858 & $0.106^{\star}$ \\
\hline C4 & $0.3741(6)$ & $0.1246(4)$ & $0.9900(3)$ & $0.036(2)$ \\
\hline C5 & $0.3961(6)$ & $0.0710(4)$ & $0.9546(3)$ & $0.0379(19)$ \\
\hline H5 & 0.4189 & 0.0333 & 0.9746 & $0.045^{\star}$ \\
\hline C6 & $0.3840(6)$ & $0.0746(4)$ & $0.8907(3)$ & $0.038(2)$ \\
\hline H6 & 0.3998 & 0.0383 & 0.8684 & $0.045^{\star}$ \\
\hline C7 & $0.3300(6)$ & $0.1778(4)$ & $0.8910(3)$ & $0.043(2)$ \\
\hline $\mathrm{H} 7$ & 0.3075 & 0.2145 & 0.8692 & $0.051^{\star}$ \\
\hline C8 & $0.3402(6)$ & $0.1785(4)$ & $0.9555(3)$ & $0.037(2)$ \\
\hline $\mathrm{H} 8$ & 0.3242 & 0.2155 & 0.9766 & $0.044^{*}$ \\
\hline C9 & $0.3377(6)$ & $0.1771(4)$ & $0.6074(3)$ & $0.039(2)$ \\
\hline $\mathrm{H} 9 \mathrm{~A}$ & 0.3617 & 0.2136 & 0.6291 & $0.046^{\star}$ \\
\hline C10 & $0.3274(6)$ & $0.1803(4)$ & $0.5429(3)$ & $0.037(2)$ \\
\hline $\mathrm{H} 10$ & 0.3438 & 0.2176 & 0.5224 & $0.044^{\star}$ \\
\hline C11 & $0.2917(6)$ & $0.1263(4)$ & $0.5088(3)$ & $0.0305(18)$ \\
\hline C12 & $0.2710(5)$ & $0.0711(4)$ & $0.5439(3)$ & $0.036(2)$ \\
\hline H12 & 0.2500 & 0.0334 & 0.5233 & $0.043^{\star}$ \\
\hline C13 & $0.2822(6)$ & $0.0736(4)$ & $0.6077(4)$ & $0.037(2)$ \\
\hline H13 & 0.2654 & 0.0372 & 0.6296 & $0.044^{\star}$ \\
\hline C14 & $0.2816(6)$ & $0.1234(5)$ & $0.4402(3)$ & $0.038(2)$ \\
\hline C15 & $0.2441(8)$ & $0.0909(7)$ & $0.3467(6)$ & $0.090(5)$ \\
\hline H15 & 0.2210 & 0.0662 & 0.3118 & $0.108^{\star}$ \\
\hline C16 & $0.2462(7)$ & $0.0738(5)$ & $0.4045(5)$ & $0.055(3)$ \\
\hline H16 & 0.2267 & 0.0340 & 0.4190 & $0.066^{\star}$ \\
\hline C17 & $0.0026(6)$ & $0.1761(4)$ & $0.6096(4)$ & $0.041(2)$ \\
\hline H17 & 0.0248 & 0.2125 & 0.6322 & 0.049 * \\
\hline C18 & $-0.0065(6)$ & $0.1789(4)$ & $0.5453(3)$ & $0.041(2)$ \\
\hline H18 & 0.0083 & 0.2167 & 0.5251 & $0.049^{\star}$ \\
\hline C19 & $-0.0379(5)$ & $0.1251(4)$ & $0.5100(3)$ & $0.033(2)$ \\
\hline $\mathrm{C} 20$ & $-0.0630(6)$ & $0.0714(4)$ & $0.5443(4)$ & $0.046(2)$ \\
\hline
\end{tabular}

Table 2 (continued)

\begin{tabular}{lrrrr}
\hline Atom & $\boldsymbol{x}$ & $\boldsymbol{y}$ & $\boldsymbol{z}$ & $\boldsymbol{U}_{\text {iso }}{ }^{*} / \boldsymbol{U}_{\text {eq }}$ \\
\hline $\mathrm{H} 20$ & -0.0877 & 0.0346 & 0.5233 & $0.056^{*}$ \\
$\mathrm{C} 21$ & $-0.0515(6)$ & $0.0727(4)$ & $0.6077(4)$ & $0.040(2)$ \\
$\mathrm{H} 21$ & -0.0673 & 0.0360 & 0.6294 & $0.048^{*}$ \\
$\mathrm{C} 22$ & $-0.0511(7)$ & $0.1236(5)$ & $0.4389(4)$ & $0.047(2)$ \\
$\mathrm{C} 23$ & $-0.0859(10)$ & $0.0899(7)$ & $0.3472(5)$ & $0.088(5)$ \\
$\mathrm{H} 23$ & -0.1051 & 0.0633 & 0.3131 & $0.106^{*}$ \\
$\mathrm{C} 24$ & $-0.0866(6)$ & $0.0746(5)$ & $0.4046(4)$ & $0.050(3)$ \\
$\mathrm{H} 24$ & -0.1085 & 0.0355 & 0.4197 & $0.059^{*}$ \\
\hline
\end{tabular}

The asymmetric unit of the title structure contains one and a half $\mathrm{Cd}(\mathrm{II})$ cation three chlorine anions distributed over four sites and three 4-(1H-pyrazol-3-yl)-pyridine molecules as bridging ligands to construct a new coordination polymer. The cadmium cation is six-coordinated by four chlorido ligands and two pyridine ligands. The $\mathrm{Cd} 1-\mathrm{N}$ bond lengths is 2.320(5) $\AA$. The $\mathrm{Cd} 1-\mathrm{Cl}$ bond lengths are 2.6859(18) $\AA$ and 2.6824(18) $\AA$, respectively. The bond angles of $\mathrm{Cl}-$ $\mathrm{Cd} 1-\mathrm{Cl}$ are in the range of $89.08(5)^{\circ}$ to $179.93(7)^{\circ}$. In addition, there are intermolecular hydrogen bonds $\mathrm{d}(\mathrm{N}(9)-$ $\mathrm{H}(9) \cdots \mathrm{Cl}(4) \# 3=2.78 \AA, \quad \mathrm{d}(\mathrm{N}(4)-\mathrm{H}(4) \cdots \mathrm{Cl}(4) \# 4=2.76 \AA$, $\mathrm{d}(\mathrm{N}(3)-\mathrm{H}(3) \cdots \mathrm{Cl}(2) \# 4=2.77$ Å. Symmetry codes: $\# 3-x+1 / 2$, $-y+1 / 2,-z+2$; \#4 $-x+1 / 2,-y+1 / 2,-z+1$. Overall, the hydrogen bonds give rise to a 3D network.

\section{References}

1. Bruker. FRAMBO and SAINT. Bruker AXS Inc., Madison, Wisconsin, USA (2002).

2. Sheldrick, G. M.: A short history of SHELX. Acta Crystallogr. A64 (2008) 112-122.

3. Chae, H. K.; Pérez, D. Y. S.; Kim, J.; Go, Y. B.; Eddaoudi, M.; Matzger, A. J.; O'Keee, M.; Yaghi, O. M.: A route to high surface area, porosity and inclusion of large molecules in crystals. Nature 427 (2004) 523-527.

4. Ma, L.; Abney, C.; Lin, W.: Enantioselective catalysis with homochiral metalorganic frameworks. Chem. Soc. Rev. 38 (2009) 1248-1256.

5. Dechambenoit, P.; Long, J. R.: Microporous magnets. Chem. Soc. Rev. 40 (2011) 3249-3265.

6. Allendorf, M. D.; Bauer, C. A.; Bhakta, R. K.; Houk, R. J. T.: Luminescent metalorganic frameworks. Chem. Soc. Rev. 38 (2009) 1330-1352.

7. Li, S. H.; Han, M. L.; Liu, G. Z.; Ma, L. F.; Wang, L. Y.: Guestinduced single-crystal-to-single-crystal transformations of a new 4-connected 3D cadmium(II) metal-organic framework. RSC Adv. 5 (2015) 17588-17591.

8. Wei, W.; Wu, M. Y.; Gao, Q.; Zhang, Q. F.; Huang, Y. G.; Jiang, F. L.; Hong, M. C.: A novel supramolecular tetrahedron assembled from tetranuclear copper(i) cluster molecules via aryl embrace interactions. Inorg. Chem. 48 (2009) 420-422. 